\title{
Problem of Inconsistent and Contradictory Judgements in Pairwise Comparison Method in Sense of AHP
}

\author{
Miroslaw Kwiesielewicz ${ }^{1}$ and Ewa van Uden ${ }^{2}$ \\ ${ }^{1}$ Technical University of Gdansk \\ Faculty of Electrical and Control Engineering \\ Narutowicza 11/12, Gdansk, POLAND, 80-952 \\ Phone: +48 58 3472124, Fax: +48 583471802 \\ mkwies@ely.pg.gda.pl \\ ${ }^{2}$ Technical University of Gdansk \\ Faculty of Electrical and Control Engineering \\ Narutowicza 11/12, Gdansk, POLAND, 80-952 \\ Phone: +4858 3471225, Fax: +48 583471802 \\ cotynato@ely.pg.gda.pl
}

\begin{abstract}
Abstr act. The aim of this paper is to show the relationship between inconsistent and contradictory matrices of data obtained as a result of the pairwise comparison of factors in the sense of the Analytic Hierarchy Process. The consistency check is performed to ensure that judgements are neither random nor illogical. This paper shows that even if a matrix will pass a consistency test successfully, it can be contradictory. Moreover an algorithm of checking contradictions is proposed.
\end{abstract}

\section{Introduction}

The methodology of AHP decomposes a complex decision problem into elemental issues to create a hierarchical model. The decision variables (factors) are situated at the lowest level. The elements with similar nature are grouped at the same interim levels and the overall goal at the highest level. At each level of the hierarchy, paired comparison judgements are obtained among stimuli. After normalisation of the results from each level, the aggregation is made.

The main aim of the pairwise comparison method in the AHP is to make a ranking of $n$ given factors or alternatives. To compare the factors often a scale $\{1 / 9,1 / 8, \ldots, 1 / 2,1,2, \ldots, 8,9\}$ introduced by Saaty $[6]$ is used. The numbers from the scale above are displayed in the matrix of judgement $\mathrm{R}=\left|\mathrm{r}_{i j}\right|_{n x n}$ expressing a relative significance of the factors $F_{i}$ and $F_{j}$.

Matrix $\mathrm{R}$ is said to be reciprocal if $r_{i j}=1 / r_{j i}$ for all $i, j=1,2, \ldots, n$ and $r_{i j}>0$.

The matrix $\mathrm{R}$ is said to be consistent if $r_{i j} r_{j k}=r_{i k}$ for all $i, j, k=1,2, \ldots, n$.

There are some reasons of having inconsistent data, namely the mistakes of a person providing his or her opinions or using 9-point semantic scale [5]. To show this phenomenon assume the following exemplary judgement matrix: 


$$
R=\left[\begin{array}{ccc}
1 & 3 & 5 \\
1 / 3 & 1 & - \\
1 / 5 & - & 1
\end{array}\right]
$$

To keep the consistency the numbers $R(2,3)=5 / 3$ and $R(3,2)=3 / 5$ should be placed in the empty entries respectively. These numbers however do not belong to assumed scale! To avoid such a situation, one can use a scale containing only the multiple of one number e.g. 2 . A scale constructed in this way would take a form: $\{\ldots 1 / 8,1 / 4,1 / 2,1,2,4,8 \ldots\}$. A quantity of numbers appearing on the scale would depend on complexity of the problem. A lot of authors discuss the problems of scale e.g. Finan and Hurley [3]. A reasonable requirement is to make inconsistencies as few as possible. The most often approach is to calculate a consistency index according to the formula $[6,7]$ :

$$
C . I .=\frac{\lambda_{\max }-n}{n-1}
$$

where $\lambda_{\max }$ denotes the maximal eigenvalue of matrix $R$. When matrix $R$ is consistent then $\lambda_{\max }=n$ and C.I. $=0$.

It's obvious that C.I. should be as close as possible to zero, but till now it is not said precisely what values are permissible. The standard procedure allows to accept a set of judgements when C.I. is not bigger than $1 / 10$ of the mean consistency index of randomly generated matrices. The mean consistency for indices matrices of size $3 \times 3$ to $9 \times 9$, proposed by Saaty and Vargas [7] are shown in Table 1.

Table 1. Mean consistency index for randomly generated matrices.

\begin{tabular}{cc}
$\begin{array}{c}\text { Matrix size } \\
(\mathrm{n})\end{array}$ & $\begin{array}{c}\text { Mean consistency } \\
\text { C.I. }\end{array}$ \\
\hline 3 & 0.5381 \\
4 & 0.8832 \\
5 & 1.1045 \\
6 & 1.2525 \\
7 & 1.3334 \\
8 & 1.4217 \\
9 & 1.4457 \\
\hline
\end{tabular}

Karpetrovic and Rosenbloom [4] give some examples when the comparisons are neither random nor illogical but they fail the consistency test.

This paper separates a subset of so-called contradictory matrices from inconsistent matrices and shows that such matrices may pass the consistency test and data may be acceptable. At the end an algorithm for checking contradictions is presented and some numerical examples are included. 


\section{Basic Concepts}

Let us introduce the following definition of contradictory judgement matrix, based on the transitivity rule.

Definition 1

The matrix $\mathrm{R}$ is called contradictory if there exists $i, j, k: 1,2, \ldots, n$ such that any of the detailed below cases hold:

$$
\begin{aligned}
& r_{i j}>1 \wedge r_{i k}<1 \wedge r_{j k}>1 \\
& r_{i j}<1 \wedge r_{i k}>1 \wedge r_{j k}<1 \\
& r_{i j}=1 \wedge r_{i k}>1 \wedge r_{j k}<1 \\
& r_{i j}=1 \wedge r_{i k}<1 \wedge r_{j k}>1 \\
& r_{i j}=1 \wedge r_{i k}=1 \wedge r_{j k}<1 \\
& r_{i j}=1 \wedge r_{i k}=1 \wedge r_{j k}>1 .
\end{aligned}
$$

Denoting: >> - "better", <<-"worst", = - "equal", it is equivalent to contradictory judgements concerning factors $F_{i}, F_{j}, F_{k}$ :

$$
\begin{aligned}
& F_{i} \gg F_{j} \text { and } F_{i} \ll F_{k} \text { and } F_{k} \ll F_{j} \\
& F_{i} \ll F_{j} \text { and } F_{i} \gg F_{k} \text { and } F_{k} \ll F_{j} \\
& F_{i}=F_{j} \text { and } F_{i} \gg F_{k} \text { and } F_{k} \ll F_{j} \\
& F_{i}=F_{j} \text { and } F_{i} \ll F_{k} \text { and } F_{k} \gg F_{j} \\
& F_{i}=F_{j} \text { and } F_{i}=F_{k} \text { and } F_{k} \ll F_{j} \\
& F_{i}=F_{j} \text { and } F_{i}=F_{k} \text { and } F_{k} \gg F_{j} .
\end{aligned}
$$

It's easy to notice that in this case a transitivity rule does not hold.

An example of a matrix with acceptable C.I., but which is contradictory is:

$$
\mathrm{R}=\left[\begin{array}{cccccccc}
1 & 2 & 1 / 2 & 2 & 1 / 2 & 2 & 1 / 2 & 2 \\
1 / 2 & 1 & 4 & 1 & 1 / 4 & 1 & 1 / 4 & 1 \\
2 & 1 / 4 & 1 & 4 & 1 & 4 & 1 & 4 \\
1 / 2 & 1 & 1 / 4 & 1 & 1 / 4 & 1 & 1 / 4 & 1 \\
2 & 4 & 1 & 4 & 1 & 4 & 1 & 4 \\
1 / 2 & 1 & 1 / 4 & 1 & 1 / 4 & 1 & 1 / 4 & 1 \\
2 & 4 & 1 & 4 & 1 & 4 & 1 & 4 \\
1 / 2 & 1 & 1 / 4 & 1 & 1 / 4 & 1 & 1 / 4 & 1
\end{array}\right]
$$


The consistency index for this matrix is equal to C.I. $=0.07252$. Moreover, note that when the entries $\mathrm{R}(2,3)$ and $\mathrm{R}(3,2)$ are replaced, the fully consistent matrix in the sense of the consistency index C.I. (1) is obtained. Analysing this matrix one may notice that $\mathrm{R}(1,2)=2, \mathrm{R}(1,3)=1 / 2$. So, one may say that factor $F_{2}$ is better then $F_{1}$, factor $F_{1}$ is better then $F_{3}$ and it would be natural to expect that $F_{2}$ were better than $F_{3}$, but $\mathrm{R}(2,3)=4$, what means that $F_{3}$ is better than $F_{2}$.

Studying this example another remark may be made. Intuitively one could expect that contradictory is the extreme form of inconsistency, but it is not true. This example shows that a matrix may be very close to perfect consistency, but it's possible to find some contradicts in the judgements. Graphically the structure of comparison matrices may be presented as shown in Fig.1.

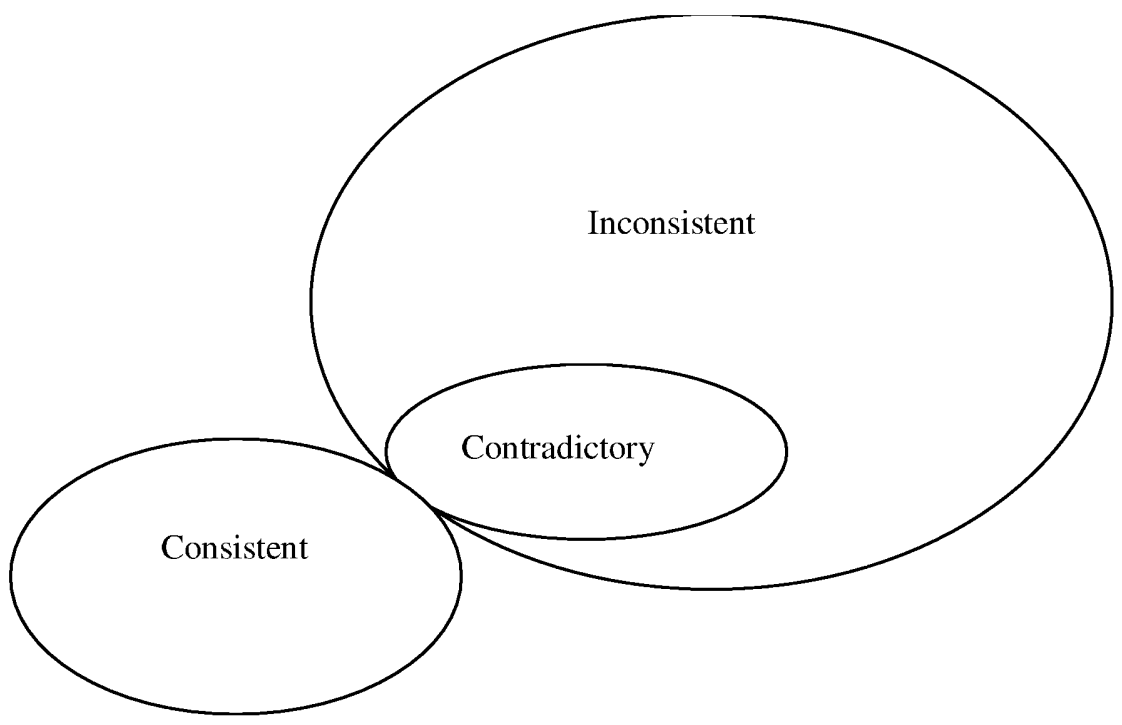

Fig. 1. Structure of the set of comparison matrice

The last task undertook by authors is to create an effective algorithm testing the contradictory. The proposal may be formulated in the following way:

FOR $(i, j, k=1$ AND $i \neq j \neq k)$ TO $n$ DO

IF $\log r_{i j} \cdot \log r_{i k} \leq 0$ AND $\log r_{i k} \cdot \log r_{j k}<0$

THEN STOP - CONTRADICTORY

ELSE 
IF $\log r_{i j}=0$ AND $\log r_{i k}=0$ AND $\log r_{j k} \neq 0$

\section{THEN STOP - CONTRADICTORY}

\section{ELSE}

OK.

\section{Calculation example}

Let us assume that an expert intended to create the following matrix of judgements comparing four factors $F_{1}, F_{2}, F_{3}, F_{4}$ :

$$
A=\left[\begin{array}{cccc}
1 & 4 & 8 & 2 \\
1 / 4 & 1 & 2 & 1 / 8 \\
1 / 8 & 1 / 2 & 1 & 1 / 9 \\
1 / 2 & 8 & 9 & 1
\end{array}\right],
$$

but by mistake he replaced the entries $a_{13}$ and $a_{31}$. In such a case his matrix has a form:

$$
\mathrm{B}=\left[\begin{array}{cccc}
1 & 4 & 1 / 8 & 2 \\
1 / 4 & 1 & 2 & 1 / 8 \\
8 & 1 / 2 & 1 & 1 / 9 \\
1 / 2 & 8 & 9 & 1
\end{array}\right]
$$

The ranking obtained from the matrix $\mathrm{A}$ is $F_{1} \gg F_{4} \gg F_{2} \gg F_{3}$, whereas the matrix B gives the ranking: $F_{4} \gg F_{1} \gg F_{3} \gg F_{2}$. It is easy to see that the "cost" of his mistake is high, in the sense that obtained ranking is quite different from the correct one. If he applied the algorithm from chapter 2 to matrix $\mathrm{B}$, he would notice that $\log b_{12} \cdot \log b_{13}<0$ and $\log b_{13} \cdot \log b_{23}<0$, what would be a hint for him that he made a mistake filling the entries of matrix $\mathrm{B}$.

In the matrix $\mathrm{B}$ one can observe the situation from definition 1, formulae (2), namely $b_{12}>1 \wedge b_{13}<1 \wedge b_{23}>1$. The consequence of the opinions expressed in matrix $\mathrm{B}$ is that factor $F_{1}$ is better than $F_{2}$, the factor $F_{2}$ is better than $F_{3}$, but the factor $F_{1}$ is worst than $F_{3}$, what obviously gives a contradiction. The algorithm created in the previous chapter allows not only to state but also localising the mistakes that can appear in judgements.

\section{Conclusions}

In this paper a new approach to consistency, inconsistency and contradictory of judgement matrices obtained as a result of the pairwise comparisons in the AHP was introduced. The method can be easily applied for the case with many experts - it is logical to check their judgements independently and to exclude these from them, who provided contradictory opinions. 
The approach presented can be extended for fuzzy approaches to the problem considered, [1,2], i.e. for the fuzzy case, contradictory in the opinion of authors, should be checked at least for the modal values.

It is necessary to stress that the consistency test is not sufficient for statement of a fact whether a set of judgements should be accepted or rejected. Therefore an additional contradictory test should be made. Without such a test, it is possible to obtain absolutely arbitrary ranking as it was shown in calculation example. Moreover, the algorithm allows localising the contradictions in judgements, so they can be easily corrected.

\section{References}

1. Buckley, J.J.: Fuzzy Hierarchical Analysis. Fuzzy Sets and Systems, Vol. 17 (1985) 233-247.

2. Buckley, J.J.: Fuzzy Hierarchical Analysis Revisited. Proc. Eight International Fuzzy Systems Association World Congress, 1999 August 17-20, Taipei, Taiwan ROC.

3. Finan, J.S., Hurley, W.J.: Transitive Calibration of the AHP Verbal Scale. European Journal of Operational Research, Vol. 112 (1999) 367-372.

4. Karpetrovic, S., Rosenbloom, E.S.: A Quality Control Approach to Consistency Paradoxes in AHP. European Journal of Operational Research, Vol. 119 (1999) 704-718.

5. Murphy, C.K.: Limits on the Analytic Hierarchy Process from its Consistency Index. European Journal of Operational Research, Vol. 65 (1993) 138-139.

6. Saaty T.L. The Analytic Hierarchy Process, Mac Grew Hill (1980)

7. Saaty, T., Vargas, L.: Comparison of Eigenvalue, Logarithmic Least Squares and Least Squares Methods in Estimating Ratios. Mathematical Modelling, Vol. 5 (1984) 309-324. 